\title{
WEAK CONVERGENCE OF A HYBRID ITERATIVE SCHEME WITH ERRORS FOR EQUILIBRIUM PROBLEMS AND COMMON FIXED POINT PROBLEMS
}

\author{
SEUng-Hyun KIm ${ }^{a}$ AND Byung-Soo LeE ${ }^{\text {b,* }}$
}

\begin{abstract}
In this paper, we consider, under a hybrid iterative scheme with errors, a weak convergence theorem to a common element of the set of a finite family of asymptotically $k$-strictly pseudo-contractive mappings and a solution set of an equilibrium problem for a given bifunction, which is the approximation version of the corresponding results of Kumam et al. [1].
\end{abstract}

\section{Introduction and Preliminaries}

The equilibrium problems were introduced by Blum and Oettli [2] in 1994. Numerous problems in applied sciences, for example optimization problems, saddle point problems, variational inequality problems and Nash equilibria in noncooperative games, are reduced to find a solution of the following equilibrium problem $[2,3]$; finding $x \in C$ such that

$$
\phi(x, y) \geq 0, \forall y \in C,
$$

where $\phi: C \times C \rightarrow \mathbb{R}$ is a bifunction.

On the other hand, the problem of finding a common fixed point of a family of mappings is a classical problem in nonlinear analysis. Finding an optimal point in the set of common fixed points of a family of mappings is a task that occurs frequently in various areas of mathematical sciences and engineering. For example, the convex feasibility problem reduces to finding a point of the set of common fixed points of a family of nonexpansive mappings [4].

Received by the editors April 10, 2014. Revised June 15, 2014. Accepted June 19, 2014. 2010 Mathematics Subject Classification. 47H09, 47H10.

Key words and phrases. equilibrium problems, fixed point problems, asymptotically $k$-strictly pseudo-contractive mappings, hybrid iterative scheme.

${ }^{*}$ Corresponding author. 
In 2009, Qin et al. considered the following weak convergence theorem to a common fixed point of a finite family of asymptotically $k$-strictly pseudo-contractive mappings under a hybrid iterative scheme.

Theorem $1.1([5])$. Assume the following conditions;

(1) $C$ is a closed convex subset of a Hilbert space $H$,

(2) $T_{i}: C \rightarrow C$ is an asymptotically $k_{i}$-strictly pseudo-contractive mapping, where $1 \leq i \leq N$ for some natural number $N$ and $0 \leq k_{i}<1$,

(3) $\left\{k_{n, i}\right\}$ is a sequence in $[1, \infty)$ such that $\sum_{n=0}^{\infty}\left(k_{n, i}-1\right)<\infty$,

(4) $k=\max \left\{k_{i}: 1 \leq i \leq N\right\}$ and

(5) $\left\{k_{n}\right\}$ is a sequence defined by $k_{n}=\max \left\{k_{n, i}: 1 \leq i \leq N\right\}$ for $n \in \mathbb{N}$.

Assume that $F:=\left(\bigcap_{i=1}^{N} F\left(T_{i}\right)\right) \neq \emptyset$. For any $x_{0} \in C$, let $\left\{x_{n}\right\}$ be a sequence generated by

$$
x_{n}=a_{n-1} x_{n-1}+\left(1-a_{n-1}\right) T_{i(n)}^{h(n)} x_{n-1}, \quad \forall n \geq 0,
$$

where $\left\{a_{n}\right\}$ is a sequence in $(0,1)$ such that $k+\varepsilon \leq a_{n} \leq 1-\varepsilon$ for some $\varepsilon \in(0,1)$ and $n=(h-1) N+i(n \geq 1)$, where $i=i(n) \in\{1,2, \cdots, N\}, h=h(n) \geq 1$ is a positive integer and $h(n) \rightarrow \infty$ as $n \rightarrow \infty$. Then $\left\{x_{n}\right\}$ converges weakly to an element of $F$.

The existence of solutions of equilibrium problems and common fixed points of finite mappings are very important in nonlinear analysis with applications. Moreover, to find the intersection of solution sets of equilibrium problems and common fixed points of finite mappings and to apply the intersection are also important. Recently, there have been a few works for the intersection of the two sets to be the set of weakly convergent points of two given sequences in Hilbert spaces.

In 2000, Kumam et al. considered the following weak convergence theorem to a given common element of the set of common fixed points of a finite family of asymptotically $k$-strictly pseudo-contractive mappings and a solution set of an equilibrium problem for a given bifunction under a hybrid iterative scheme.

Theorem $1.2([1])$. Assume the conditions (1)-(5) in Theorem 1.1. Let $\phi: C \times C \rightarrow$ $\mathbb{R}$ be a bifunction satisfying the followings;

(A1) $\phi(x, x)=0, \forall x \in C$;

(A2) $\phi$ is monotone, i.e., $\phi(x, y)+\phi(y, x) \leq 0$ for any $x, y \in C$; 
(A3) $\phi$ is upper-hemicontinuous, i.e., for each $x, y, z \in C$,

$$
\lim \sup _{t \rightarrow 0+} \phi(t z+(1-t) x, y) \leq \phi(x, y)
$$

(A4) $\phi(x, \cdot)$ is convex and lower semicontinuous for each $x \in C$.

Assume that $F:=\left(\bigcap_{i=1}^{N} F\left(T_{i}\right)\right) \bigcap E P(\phi) \neq \emptyset$, where $E P(\phi)$ is a set of solutions of equilibrium problem (1.1). For any $x_{0} \in C$, let $\left\{x_{n}\right\}$ and $\left\{v_{n}\right\}$ be sequences generated by

$$
\left\{\begin{array}{l}
\phi\left(v_{n-1}, y\right)+\frac{1}{r_{n-1}}\left\langle y-v_{n-1}, v_{n-1}-x_{n-1}\right\rangle \geq 0, \quad \forall y \in C, \\
x_{n}=a_{n-1} v_{n-1}+\left(1-a_{n-1}\right) T_{i(n)}^{h(n)} v_{n-1}, \quad \forall n \geq 0,
\end{array}\right.
$$

where $n=(h-1) N+i(n \geq 1), i=i(n) \in\{1,2, \cdots, N\}, h=h(n) \geq 1$ is a positive integer and $h(n) \rightarrow \infty$ as $n \rightarrow \infty$. Let $\left\{a_{n}\right\}$ and $\left\{r_{n}\right\}$ satisfy the following conditions:

(1) $\left\{a_{n}\right\} \subset[\alpha, \beta]$, for some $\alpha, \beta \in(k, 1)$ and

(2) $\left\{r_{n}\right\} \subset(0, \infty)$ and $\lim _{n \rightarrow \infty} \inf r_{n}>0$.

Then $\left\{x_{n}\right\}$ and $\left\{v_{n}\right\}$ converges weakly to an element of $F$.

On the other hand, the fixed point iterative scheme with errors was introduced by Liu [6]. The idea of considering fixed point iterative scheme problems with errors which comes from practical numerical computation usually concerns the approximation fixed point and is related to the stability of fixed point iterative schemes. The idea of considering iterative scheme procedures with errors leads to finding the approximate solution to equilibrium problems. In 2005, Combettes and Hirstoga [3] introduced an iterative scheme for a problem of finding best approximate solutions to equilibrium problem and proved the strong convergence result.

In this paper, we consider, under a hybrid iterative scheme with errors, a weak convergence theorem to a common element of the set of a finite family of asymptotically $k$-strictly pseudo-contractive mappings and a solution set of an equilibrium problem for a given bifunction, which is the approximation version of the corresponding results of Kumam et al. [1].

The following results will be needed in the main result.

Lemma 1.1 $([7,8])$. Let $H$ be a real Hilbert space. There hold the following identities

(i) $\|x-y\|^{2}=\|x\|^{2}-\|y\|^{2}-2\langle x-y, y\rangle, \forall x, y \in H$,

(ii) $\|a x+b y+c z\|^{2}=a\|x\|^{2}+b\|y\|^{2}+c\|z\|^{2}-a b\|x-y\|^{2}-b c\|y-z\|^{2}-c a\|z-x\|^{2}$, $\forall x, y \in H$, where $a, b, c \in[0,1]$ with $a+b+c=1$, 
(iii) If $\left\{x_{n}\right\}$ is a sequence in $H$ weakly converging to $z$, then

$$
\lim _{n \rightarrow \infty} \sup \left\|x_{n}-y\right\|^{2}=\lim _{n \rightarrow \infty} \sup \left\|x_{n}-z\right\|^{2}+\|z-y\|^{2}, \quad \forall y \in H .
$$

Lemma $1.2([3])$. Assume that $\phi: C \times C \rightarrow \mathbb{R}$ satisfies (A1)-(A4). For $r>0$ and $x \in H$, define a mapping $S_{r}: H \rightarrow C$ as follows;

$$
S_{r}(x)=\left\{z \in C: \phi(z, y)+\frac{1}{r}\langle y-z, z-x\rangle \geq 0, \forall y \in C\right\},
$$

for all $z \in H$. Then the following hold;

(i) $S_{r}$ is single-valued;

(ii) $S_{r}$ is firmly nonexpansive, i.e., for any $x, y \in H$,

$$
\left\|S_{r} x-S_{r} y\right\|^{2} \leq\left\langle S_{r} x-S_{r} y, x-y\right\rangle ;
$$

(iii) $F\left(S_{r}\right)=E P(\phi)$;

(iv) $\operatorname{EP}(\phi)$ is closed and convex.

\section{Main Results}

Definition 2.1. A mapping $T: C \rightarrow C$ is said to be asymptotically $k$-strictly pseudo-contractive if there exist a sequence $\left\{k_{n}\right\} \subset[1, \infty)$ with $\lim _{n \rightarrow \infty} k_{n}=1$ and $k \in[0,1)$ such that

$\left\|T^{n} x-T^{n} y\right\|^{2} \leq k_{n}^{2}\|x-y\|^{2}+k\left\|\left(I-T^{n}\right) x-\left(I-T^{n}\right) y\right\|^{2}, \quad \forall x, y \in C$ and $n \in \mathbb{N}$.

The following proposition by Osilike and Igbokwe [8] was considered by using infinite terms of the given sequences based on Lemma 1 in [9], but our proof is considered by using only finite terms of the given sequences based on the basic concepts of limit superior and limit inferior.

Proposition 2.1. Let $\left\{a_{n}\right\},\left\{c_{n}\right\}$ and $\left\{\delta_{n}\right\}$ be nonnegative real sequences satisfying the following condition:

$$
a_{n+1} \leq\left(1+\delta_{n}\right) a_{n}+c_{n}, \forall n \in \mathbb{N} .
$$

If $\sum_{n=1}^{\infty} \delta_{n}<\infty$ and $\sum_{n=1}^{\infty} c_{n}<\infty$, then $\lim _{n \rightarrow \infty} a_{n}$ exists.

Proof. Consider

$$
\begin{aligned}
a_{n+m} & \leq\left(1+\delta_{n+m-1}\right) a_{n+m-1}+c_{n+m-1} \\
& \leq\left(1+\delta_{n+m-1}\right)\left\{\left(1+\delta_{n+m-2}\right) a_{n+m-2}+c_{n+m-2}\right\}+c_{n+m-1}
\end{aligned}
$$




$$
\begin{aligned}
& =\left(1+\delta_{n+m-1}\right)\left(1+\delta_{n+m-2}\right) a_{n+m-2}+\left(1+\delta_{n+m-1}\right) c_{n+m-2}+c_{n+m-1} \\
& \leq \cdots \\
& \leq \prod_{j=n}^{n+m-1}\left(1+\delta_{j}\right) a_{n}+\sum_{j=n}^{n+m-1}\left\{c_{j} \prod_{j=n+1}^{n+m-1}\left(1+\delta_{j}\right)\right\} \\
& \leq e^{\sum_{j=n}^{n+m-1} \delta_{j}} a_{n}+\sum_{j=n}^{n+m-1} c_{j} e^{\sum_{j=n}^{n+m-1} \delta_{j}} .
\end{aligned}
$$

Thus $\limsup _{m \rightarrow \infty} a_{m} \leq e^{\sum_{j=n}^{\infty} \delta_{j}} a_{n}+\sum_{j=n}^{\infty} c_{j} e^{\sum_{j=n}^{\infty} \delta_{j}}$. Since $\sum_{n=1}^{\infty} \delta_{n}<\infty$ and $\sum_{n=1}^{\infty} c_{n}<\infty$ for any $\varepsilon>0$, take $N \in \mathbb{N}$ such that $\sum_{j=n}^{\infty} \delta_{j}<\varepsilon$ and $\sum_{j=n}^{\infty} c_{j}<\varepsilon$, for $n \geq N$. Thus, $\limsup _{m \rightarrow \infty} a_{m} \leq e^{\varepsilon} a_{n}+\varepsilon e^{\varepsilon}$. Letting $\varepsilon \rightarrow 0$, we have the wanted result. Hence $\limsup _{m \rightarrow \infty} a_{m} \leq \liminf _{n \rightarrow \infty} a_{n}$, which shows the existence of $\lim _{n \rightarrow \infty} a_{n}$.

Putting $\delta_{n}=0(\forall n \in \mathbb{N})$, we have the following known lemma as a corollary;

Lemma 2.1 ([9]). Let $\left\{a_{n}\right\}$ and $\left\{b_{n}\right\}$ be nonnegative real sequences satisfying the following condition:

$$
a_{n+1} \leq a_{n}+b_{n}, \quad \forall n \in \mathbb{N} .
$$

If $\sum_{n=1}^{\infty} b_{n}<\infty$, then $\lim _{n \rightarrow \infty} a_{n}$ exists.

Now, we prove our main result.

Theorem 2.1. Assume the conditions (1)-(5) in Theorem 1.1. Let $\phi: C \times C \rightarrow \mathbb{R}$ be a bifunction satisfying (A1)-(A4). Assume that $F:=\left(\bigcap_{i=1}^{N} F\left(T_{i}\right)\right) \bigcap E P(\phi) \neq \emptyset$. For any $x_{0} \in C$, let $\left\{x_{n}\right\}$ and $\left\{v_{n}\right\}$ be sequences generated by

$$
\left\{\begin{array}{l}
\phi\left(v_{n-1}, y\right)+\frac{1}{r_{n-1}}\left\langle y-v_{n-1}, v_{n-1}-x_{n-1}\right\rangle \geq 0, \quad \forall y \in C, \\
x_{n}=a_{n-1} v_{n-1}+b_{n-1} T_{i(n)}^{h(n)} v_{n-1}+c_{n-1} u_{n-1}, \quad \forall n \geq 0 ;
\end{array}\right.
$$

where $\left\{a_{n}\right\},\left\{b_{n}\right\}$ and $\left\{c_{n}\right\}$ are sequences in $[0,1)$ such that $a_{n}+b_{n}+c_{n}=1$, $a_{n} \geq k+\varepsilon, b_{n} \geq \varepsilon$ for some $\varepsilon \in(0,1), \sum_{n=1}^{\infty} c_{n}<\infty,\left\{u_{n}\right\}$ is a bounded sequence in $C,\left\{r_{n}\right\}$ is a sequence in $(0, \infty)$ such that $\lim _{n \rightarrow \infty} \inf r_{n}>0$ and $n=(h-1) N+i(n \geq 1)$, where $i=i(n) \in\{1,2, \cdots, N\}, h=h(n) \geq 1$ is a positive integer and $h(n) \rightarrow \infty$ as $n \rightarrow \infty$. Then $\left\{x_{n}\right\}$ and $\left\{v_{n}\right\}$ converges weakly to an element of $F$. 
Proof. Let $p \in F$. From (2.1) and Lemma 1.2, we have $v_{n-1}=S_{r_{n-1}} x_{n-1}$ and

$$
\begin{aligned}
\left\|v_{n-1}-p\right\| & =\left\|S_{r_{n-1}} x_{n-1}-S_{r_{n-1}} p\right\| \\
& \leq\left\|x_{n-1}-p\right\|, \quad \forall n \geq 0 .
\end{aligned}
$$

From (2.1) and Lemma 1.1(ii),

$$
\begin{aligned}
& \left\|x_{n}-p\right\|^{2} \\
& =\left\|a_{n-1} v_{n-1}+b_{n-1} T_{i(n)}^{h(n)} v_{n-1}+c_{n-1} u_{n-1}-\left(a_{n-1}+b_{n-1}+c_{n-1}\right) p\right\|^{2} \\
& =\left\|a_{n-1}\left(v_{n-1}-p\right)+b_{n-1}\left(T_{i(n)}^{h(n)} v_{n-1}-p\right)+c_{n-1}\left(u_{n-1}-p\right)\right\|^{2} \\
& =a_{n-1}\left\|v_{n-1}-p\right\|^{2}+b_{n-1}\left\|T_{i(n)}^{h(n)} v_{n-1}-p\right\|^{2}+c_{n-1}\left\|u_{n-1}-p\right\|^{2} \\
& -a_{n-1} b_{n-1}\left\|v_{n-1}-T_{i(n)}^{h(n)} v_{n-1}\right\|^{2}-b_{n-1} c_{n-1}\left\|T_{i(n)}^{h(n)} v_{n-1}-u_{n-1}\right\|^{2} \\
& -c_{n-1} a_{n-1}\left\|u_{n-1}-v_{n-1}\right\|^{2} \\
& \leq a_{n-1}\left\|v_{n-1}-p\right\|^{2}+b_{n-1}\left\|T_{i(n)}^{h(n)} v_{n-1}-p\right\|^{2}+c_{n-1}\left\|u_{n-1}-p\right\|^{2} \\
& -a_{n-1} b_{n-1}\left\|T_{i(n)}^{h(n)} v_{n-1}-v_{n-1}\right\|^{2} \\
& =a_{n-1}\left\|v_{n-1}-p\right\|^{2}+b_{n-1}\left\|T_{i(n)}^{h(n)} v_{n-1}-T_{i(n)}^{h(n)} p\right\|^{2}+c_{n-1}\left\|u_{n-1}-p\right\|^{2} \\
& -a_{n-1} b_{n-1}\left\|T_{i(n)}^{h(n)} v_{n-1}-v_{n-1}\right\|^{2} \\
& \leq a_{n-1}\left\|v_{n-1}-p\right\|^{2}+b_{n-1}\left\{k_{h(n)}\left\|v_{n-1}-p\right\|^{2}+k \|\left(I-T_{i(n)}^{h(n)}\right) v_{n-1}\right. \\
& \left.-\left(I-T_{i(n)}^{h(n)}\right) p \|^{2}\right\}+c_{n-1}\left\|u_{n-1}-p\right\|^{2}-a_{n-1} b_{n-1}\left\|T_{i(n)}^{h(n)} v_{n-1}-v_{n-1}\right\|^{2} \\
& =\left(a_{n-1}+b_{n-1} k_{h(n)}^{2}\right)\left\|v_{n-1}-p\right\|^{2}-b_{n-1}\left(a_{n-1}-k\right)\left\|T_{i(n)}^{h(n)} v_{n-1}-v_{n-1}\right\|^{2} \\
& +c_{n-1}\left\|u_{n-1}-p\right\|^{2} \\
& \leq\left(a_{n-1} k_{h(n)}^{2}+b_{n-1} k_{h(n)}^{2}\right)\left\|v_{n-1}-p\right\|^{2} \\
& -b_{n-1}\left(a_{n-1}-k\right)\left\|T_{i(n)}^{h(n)} v_{n-1}-v_{n-1}\right\|^{2}+c_{n-1}\left\|u_{n-1}-p\right\|^{2} \\
& \leq\left[1+\left(k_{h(n)}^{2}-1\right)\right]\left\|x_{n-1}-p\right\|^{2}+c_{n-1}\left\|u_{n-1}-p\right\|^{2} .
\end{aligned}
$$

From Proposition 2.1, $\lim _{n \rightarrow \infty}\left\|x_{n}-p\right\|$ exists. Observe (2.2) again

$$
\begin{aligned}
& b_{n-1}\left(a_{n-1}-k\right)\left\|T_{i(n)}^{h(n)} v_{n-1}-v_{n-1}\right\|^{2} \\
& \quad \leq k_{h(n)}^{2}\left\|x_{n-1}-p\right\|^{2}-\left\|x_{n}-p\right\|^{2}+c_{n-1}\left\|u_{n-1}-p\right\|^{2} .
\end{aligned}
$$

Since $a_{n} \geq k+\varepsilon, b_{n} \geq \varepsilon$ for all $n \geq 0$ and some $\varepsilon \in(0,1)$,

$$
\begin{aligned}
& \leq b_{n-1}\left(a_{n-1}-k\right)\left\|T_{i(n)}^{h(n)} v_{n-1}-v_{n-1}\right\|^{2} \\
& \leq k_{h(n)}^{2}\left\|x_{n-1}-p\right\|^{2}-\left\|x_{n}-p\right\|^{2}+c_{n-1}\left\|u_{n-1}-p\right\|^{2} .
\end{aligned}
$$


Taking the limits as $n \rightarrow \infty$, we have

$$
\lim _{n \rightarrow \infty}\left\|T_{i(n)}^{h(n)} v_{n-1}-v_{n-1}\right\|^{2}=0 .
$$

Observe that

$$
\begin{aligned}
\left\|x_{n}-v_{n-1}\right\| & =\left\|a_{n-1} v_{n-1}+b_{n-1} T_{i(n)}^{h(n)} v_{n-1}+c_{n-1} u_{n-1}-v_{n-1}\right\| \\
& =\left\|-\left(1-a_{n-1}\right) v_{n-1}+b_{n-1} T_{i(n)}^{h(n)} v_{n-1}+c_{n-1} u_{n-1}\right\| \\
& =\left\|-\left(1-a_{n-1}\right) v_{n-1}+\left(1-a_{n-1}-c_{n-1}\right) T_{i(n)}^{h(n)} v_{n-1}+c_{n-1} u_{n-1}\right\| \\
& =\left\|-\left(1-a_{n-1}\right)\left(v_{n-1}-T_{i(n)}^{h(n)} v_{n-1}\right)+c_{n-1}\left(u_{n-1}-T_{i(n)}^{h(n)} v_{n-1}\right)\right\| \\
& \leq\left(1-a_{n-1}\right)\left\|v_{n-1}-T_{i(n)}^{h(n)} v_{n-1}\right\|+c_{n-1}\left\|u_{n-1}-T_{i(n)}^{h(n)} v_{n-1}\right\| .
\end{aligned}
$$

It follows that

$$
\lim _{n \rightarrow \infty}\left\|x_{n}-v_{n-1}\right\|=0 .
$$

Since $S_{r_{n-1}}$ is firmly nonexpansive, we have

$$
\begin{aligned}
\left\|v_{n-1}-p\right\|^{2} & =\left\|S_{r_{n-1}} x_{n-1}-S_{r_{n-1}} p\right\|^{2} \\
& \leq\left\langle S_{r_{n-1}} x_{n-1}-S_{r_{n-1}} p, x_{n-1}-p\right\rangle \\
& =\left\langle v_{n-1}-p, x_{n-1}-p\right\rangle \\
& =\left\langle v_{n-1}-x_{n-1}+x_{n-1}-p, x_{n-1}-p\right\rangle \\
& =-\left\langle-\left(x_{n-1}-v_{n-1}\right)-\left(x_{n-1}-p\right), x_{n-1}-p\right\rangle \\
& =-\frac{1}{2}\left(\left\|x_{n-1}-v_{n-1}\right\|^{2}-\left\|x_{n-1}-p\right\|^{2}-\left\|v_{n-1}-p\right\|^{2}\right) \\
& =\frac{1}{2}\left(\left\|v_{n-1}-p\right\|^{2}+\left\|x_{n-1}-p\right\|^{2}-\left\|x_{n-1}-v_{n-1}\right\|^{2}\right)
\end{aligned}
$$

and hence

$$
\left\|v_{n-1}-p\right\|^{2} \leq\left\|x_{n-1}-p\right\|^{2}-\left\|x_{n-1}-v_{n-1}\right\|^{2} .
$$

Using (2.2) and (2.7), we have

$$
\begin{aligned}
\left\|x_{n}-p\right\|^{2} & \leq k_{h(n)}^{2}\left\|v_{n-1}-p\right\|^{2}+c_{n-1}\left\|u_{n-1}-p\right\|^{2} \\
& \leq k_{h(n)}^{2}\left(\left\|x_{n-1}-p\right\|^{2}-\left\|x_{n-1}-v_{n-1}\right\|^{2}\right)+c_{n-1}\left\|u_{n-1}-p\right\|^{2},
\end{aligned}
$$

hence

$$
k_{h(n)}^{2}\left\|x_{n-1}-v_{n-1}\right\|^{2} \leq k_{h(n)}^{2}\left\|x_{n-1}-p\right\|^{2}-\left\|x_{n}-p\right\|^{2}+c_{n-1}\left\|u_{n-1}-p\right\|^{2} .
$$


Since $\lim _{n \rightarrow \infty}\left\|x_{n}-p\right\|$ exists and $\lim _{n \rightarrow \infty} k_{h(n)}=1$,

$$
\lim _{n \rightarrow \infty}\left\|x_{n-1}-v_{n-1}\right\|=0 .
$$

From (2.6) and (2.8), we have

$$
\left\|v_{n}-v_{n-1}\right\| \leq\left\|v_{n}-x_{n}\right\|+\left\|x_{n}-v_{n-1}\right\| \rightarrow 0 \text { as } n \rightarrow \infty .
$$

It follows that

$$
\lim _{n \rightarrow \infty}\left\|v_{n}-v_{n+j}\right\|=0, \quad \forall j \in\{1, \cdots, N\} .
$$

Applying (2.8) and (2.9), we obtain

$$
\begin{aligned}
\left\|x_{n}-x_{n-1}\right\| & =\left\|x_{n}-v_{n}+v_{n}-v_{n-1}+v_{n-1}-x_{n-1}\right\| \\
& \leq\left\|x_{n}-v_{n}\right\|+\left\|v_{n}-v_{n-1}\right\|+\left\|v_{n-1}-x_{n-1}\right\| \rightarrow 0 \text { as } n \rightarrow \infty,
\end{aligned}
$$

which implies that $\lim _{n \rightarrow \infty}\left\|x_{n}-x_{n+j}\right\|=0, \forall j \in\{1, \cdots, N\}$. Since, for any positive integer $n>N$, it can be written as $n=(k(n)-1) N+i(n)$, where $i(n) \in\{1,2, \cdots, N\}$, observe that

$$
\begin{aligned}
\| & v_{n-1}-T_{n} v_{n-1} \| \\
\leq & \left\|v_{n-1}-T_{i(n)}^{h(n)} v_{n-1}\right\|+\left\|T_{i(n)}^{h(n)} v_{n-1}-T_{n} v_{n-1}\right\| \\
(2.11)= & \left\|v_{n-1}-T_{i(n)}^{h(n)} v_{n-1}\right\|+\left\|T_{i(n)} T_{i(n)}^{h(n)-1} v_{n-1}-T_{i(n)} v_{n-1}\right\| \\
\leq & \left\|v_{n-1}-T_{i(n)}^{h(n)} v_{n-1}\right\|+L\left\|T_{i(n)}^{h(n)-1} v_{n-1}-v_{n-1}\right\| \\
\leq & \left\|v_{n-1}-T_{i(n)}^{h(n)} v_{n-1}\right\|+L\left(\left\|T_{i(n)}^{h(n)-1} v_{n-1}-T_{i(n-N)}^{h(n)-1} v_{n-N}\right\|+\| T_{i(n-N)}^{h(n)-1} v_{n-N}\right. \\
& \left.\quad-v_{n-N-1}\|+\| v_{n-N-1}-v_{n-1} \|\right) .
\end{aligned}
$$

Since, for each $n>N, n \equiv n-N(\bmod N)$ and $n=(k(n)-1) N+i(n)$, we have $n-N=(k(n)-1) N+i(n)=(k(n-N)-1) N+i(n-N)$, that is, $i(n-N)=i(n)$, $k(n-N)=k(n)-1$. Observe that

$$
\begin{aligned}
\left\|T_{i(n)}^{h(n)-1} v_{n-1}-T_{i(n-N)}^{h(n)-1} v_{n-N}\right\| & =\left\|T_{i(n)}^{h(n)-1} v_{n-1}-T_{i(n)}^{h(n)-1} v_{n-N}\right\| \\
& \leq L\left\|v_{n-1}-v_{n-N}\right\|
\end{aligned}
$$


and

$$
\begin{aligned}
& \left\|T_{i(n-N)}^{h(n)-1} v_{n-N}-v_{n-N-1}\right\| \\
& \quad=\left\|T_{i(n-N)}^{h(n-N)} v_{n-N}-v_{n-N-1}\right\| \\
& \quad \leq\left\|T_{i(n-N)}^{h(n-N)} v_{n-N}-T_{i(n-N)}^{h(n)-N} v_{n-N-1}\right\|+\left\|T_{i(n-N)}^{h(n)-N} v_{n-N-1}-v_{n-N-1}\right\| \\
& \quad \leq L \cdot\left\|v_{n-N}-v_{n-N-1}\right\|+\left\|T_{i(n-N)}^{h(n)-N} v_{n-N-1}-v_{n-N-1}\right\| .
\end{aligned}
$$

It follows from (2.11)-(2.13) that

$$
\begin{aligned}
& \left\|v_{n-1}-T_{n} v_{n-1}\right\| \\
& \leq\left\|v_{n-1}-T_{i(n)}^{h(n)} v_{n-1}\right\|+L \cdot\left\{\left\|T_{i(n)}^{h(n)-1} v_{n-1}-T_{i(n-N)}^{h(n)-1} v_{n-N}\right\|+\| T_{i(n-N)}^{h(n)-1} v_{n-N}\right. \\
& \left.\quad-v_{n-N-1}\|+\| v_{n-N-1}-v_{n-1} \|\right\} \\
& \leq\left\|v_{n-1}-T_{i(n)}^{h(n)} v_{n-1}\right\|+L \cdot\left\{L\left\|v_{n-1}-v_{n-N}\right\|+L \cdot\left\|v_{n-N}-v_{n-N-1}\right\|\right. \\
& \left.\quad+\left\|T_{i(n-N)}^{h(n)-N} v_{n-N-1}-v_{n-N-1}\right\|+\left\|v_{n-N-1}-v_{n-1}\right\|\right\} .
\end{aligned}
$$

Applying (2.5) and (2.10) to (2.14), we obtain

$$
\lim _{n \rightarrow \infty}\left\|v_{n-1}-T_{n} v_{n-1}\right\|=0 .
$$

From (2.9) and (2.15),

$$
\begin{aligned}
\left\|v_{n}-T_{n} v_{n}\right\| & \leq\left\|v_{n}-v_{n-1}\right\|+\left\|v_{n-1}-T_{n} v_{n-1}\right\|+\left\|T_{n} v_{n-1}-T_{n} v_{n}\right\| \\
& \leq(1+L) \cdot\left\|v_{n}-v_{n-1}\right\|+\left\|v_{n-1}-T_{n} v_{n-1}\right\| \rightarrow 0 \text { as } n \rightarrow \infty .
\end{aligned}
$$

Also, we have

$$
\begin{aligned}
\left\|v_{n}-T_{n+j} v_{n}\right\| & \leq\left\|v_{n}-v_{n+j}\right\|+\left\|v_{n+j}-T_{n+j} v_{n+j}\right\|+\left\|T_{n+j} v_{n+j}-T_{n+j} v_{n}\right\| \\
& \leq(1+L) \cdot\left\|v_{n}-v_{n+j}\right\|+\left\|v_{n+j}-T_{n+j} v_{n+j}\right\| \rightarrow 0 \text { as } n \rightarrow \infty
\end{aligned}
$$

for any $j=1, \cdots, N$, which gives that

$$
\lim _{n \rightarrow \infty}\left\|v_{n}-T_{l} v_{n}\right\|=0, \quad \forall l \in\{1, \cdots, N\} .
$$

Moreover, for each $l \in\{1, \cdots, N\}$, we have

$$
\begin{aligned}
\left\|x_{n}-T_{l} x_{n}\right\| & \leq\left\|x_{n}-v_{n}\right\|+\left\|v_{n}-T_{l} v_{n}\right\|+\left\|T_{l} v_{n}-T_{l} x_{n}\right\| \\
& \leq(1+L) \cdot\left\|x_{n}-v_{n}\right\|+\left\|v_{n}-T_{l} v_{n}\right\| \rightarrow 0 \text { as } n \rightarrow \infty .
\end{aligned}
$$

Put $W\left(x_{n}\right)=\left\{x \in H: x_{n_{i}} \rightarrow x\right.$ for some subsequence $\left\{x_{n_{i}}\right\}$ of $\left.\left\{x_{n}\right\}\right\}$.

Firstly, $W\left(x_{n}\right) \neq \emptyset$. Indeed, since $\left\{x_{n}\right\}$ is bounded and $H$ is reflexive, $W\left(x_{n}\right) \neq \emptyset$. 
Secondly, we claim that

$$
W\left(x_{n}\right) \subset F .
$$

Let $w \in W\left(x_{n}\right)$ be an arbitrary element. Then there exists a subsequence $\left\{x_{n_{i}}\right\}$ of $\left\{x_{n}\right\}$ converging weakly to $w$. Applying (2.8), we can obtain that $v_{n_{i}} \rightarrow w$ as $i \rightarrow \infty$. It follows from $\lim _{n \rightarrow \infty}\left\|v_{n}-T_{l} v_{n}\right\|=0$ that $T_{l} v_{n_{i}} \rightarrow w, \forall l \in\{1, \cdots, N\}$. Let us show that $w \in E P(\phi)$. Since $v_{n}=T_{r_{n}} v_{n}$, we have

$$
\phi\left(v_{n}, y\right)+\frac{1}{r_{n}}\left\langle y-v_{n}, v_{n}-x_{n}\right\rangle \geq 0, \quad \forall y \in C .
$$

From (A2), we have

$$
\frac{1}{r_{n}}\left\langle y-v_{n}, v_{n}-x_{n}\right\rangle \geq \phi\left(y, v_{n}\right)
$$

and hence

$$
\left\langle y-v_{n_{i}}, \frac{v_{n_{i}}-x_{n_{i}}}{r_{n_{i}}}\right\rangle \geq \phi\left(y, v_{n_{i}}\right) .
$$

Since $\frac{v_{n_{i}}-x_{n_{i}}}{r_{n_{i}}} \rightarrow 0$ and $v_{n_{i}} \rightarrow w$ as $i \rightarrow \infty$, from (A4), we have

$$
\phi(y, w) \leq 0, \quad \forall y \in C .
$$

For $t \in(0,1]$ and $y \in C$, let $y_{t}=t y+(1-t) w$. Since $y \in C$ and $w \in C, y_{t} \in C$, and hence $\phi\left(y_{t}, w\right) \leq 0$. So, from (A1) and (A4),

$$
0=\phi\left(y_{t}, y_{t}\right) \leq t \phi\left(y_{t}, y\right)+(1-t) \phi\left(y_{t}, w\right) \leq t \phi\left(y_{t}, y\right)
$$

and hence $0 \leq \phi\left(y_{t}, y\right)$. From (A3), $0 \leq \phi(w, y), \forall y \in C$ and hence $w \in E P(\phi)$.

Next, we prove that $w \in\left(\bigcap_{i=1}^{N} F\left(T_{i}\right)\right)$. Suppose that $w \notin\left(\bigcap_{i=1}^{N} F\left(T_{i}\right)\right)$. Then there exists $l \in\{1, \cdots, N\}$ such that $w \notin F\left(T_{l}\right)$. From (2.16) and the Opial's condition,

$$
\begin{aligned}
\lim _{i \rightarrow \infty} \inf \left\|x_{n_{i}}-w\right\| & <\lim _{i \rightarrow \infty} \inf \left\|x_{n_{i}}-T_{l} w\right\| \\
& \leq \lim _{i \rightarrow \infty} \inf \left\{\left\|x_{n_{i}}-T_{l} x_{n_{i}}\right\|+\left\|T_{l} x_{n_{i}}-T_{l} w\right\|\right\} \\
& \leq \lim _{i \rightarrow \infty} \inf L \cdot\left\|x_{n_{i}}-w\right\|,
\end{aligned}
$$

which derives a contradiction. Hence $w \in\left(\bigcap_{i=1}^{N} F\left(T_{i}\right)\right)$.

Finally, we show that $\left\{x_{n}\right\}$ and $\left\{v_{n}\right\}$ converge weakly to an element of $F$. Indeed, it is sufficient to show that $W\left(x_{n}\right)$ is a single point set. We take $w_{1}, w_{2} \in W\left(x_{n}\right)$ arbitrarily and let $\left\{x_{n_{i}}\right\}$ and $\left\{x_{n_{j}}\right\}$ be subsequences of $\left\{x_{n}\right\}$ such that $x_{n_{i}} \rightarrow w_{1}$ and 
$x_{n_{j}} \rightarrow w_{2}$. Since $\lim _{n \rightarrow \infty}\left\|x_{n}-p\right\|$ exists for each $p \in F$ and $w_{1}, w_{2} \in F$, by Lemma 1.1 (iii), we obtain

$$
\begin{aligned}
\lim _{n \rightarrow \infty}\left\|x_{n}-w_{1}\right\|^{2} & =\lim _{j \rightarrow \infty}\left\|x_{n_{j}}-w_{1}\right\|^{2} \\
& =\lim _{j \rightarrow \infty}\left\|x_{n_{j}}-w_{2}\right\|^{2}+\left\|w_{2}-w_{1}\right\|^{2} \\
& =\lim _{i \rightarrow \infty}\left\|x_{n_{i}}-w_{2}\right\|^{2}+\left\|w_{2}-w_{1}\right\|^{2} \\
& =\lim _{i \rightarrow \infty}\left\|x_{n_{i}}-w_{1}\right\|^{2}+2\left\|w_{2}-w_{1}\right\|^{2} \\
& =\lim _{n \rightarrow \infty}\left\|x_{n}-w_{1}\right\|^{2}+2\left\|w_{2}-w_{1}\right\|^{2}
\end{aligned}
$$

Hence $w_{1}=w_{2}$, which shows that $W\left(x_{n}\right)$ is single point set.

Remark 2.1. (1) If $c_{n}=0(\forall n \in N)$ in Theorem 2.1, then we obtain Theorem 1.2. (2) If $\phi(x, y)=0(\forall x, y \in C)$ and $v_{n}=x_{n}, \forall n \in \mathbb{N}$ in (2.1), then we obtain Theorem 1.1 .

\section{REFERENCES}

1. P. Kumam, N. Petrot \& R. Wangkeeree: A hybrid iterative scheme for equilibrium problems and fixed point problems of asymptotically $k$-strict pseudo-contractions. $J$. Comp. Appl. Math. 233 (2010), 2013-2026.

2. E. Blum \& W. Oettli: From optimization and variational inequalities to equilibrium problems. Math. Student 63 (1994), 123-145.

3. P.L. Combettes \& S.A. Hirstoaga: Equilibrium programming in Hilbert spaces. $J$. Nonlinear Convex Anal. 6 (2005), 117-136.

4. H.H. Bauschke \& J.M. Borwein: On projection algorithms for solving convex feasibility problems. SIAM Rev. 38 (1996), 367-426.

5. X. Qin, Y.J. Cho, S.M. Kang \& M. Shang: A hybrid iterative scheme for asymptotically $k$-strict pseudo-contractions in Hilbert spaces. Nonlinear Anal. 70 (2009), 1902-1911.

6. L.S. Liu: Ishikawa and Mann iterative process with errors for nonlinear strongly accretive mappings in Banach spaces. J. Math. Anal. Appl. 194 (1995), 114-115.

7. G. Marino \& H.K. Xu: Weak and strong convergence theorems for strict pseudocontractions in Hilbert space. J. Math. Anal. Appl. 329 (2007), 336-346.

8. M.O. Osilike \& D.I. Igbokwe: Weak and strong convergence theorems for fixed points of pseudocontractions and solutions of monotone type operator equations. Comput. Math. Appl. 40 (2000), 559-567.

9. K.K. Tan \& H.K. Xu: Approximating fixed points of nonexpansive mappings by the Ishikawa iteration process. J. Math. Anal. Appl. 178 (1993), no. 2, 301-308. 
${ }^{a}$ Department of Mathematics, Kyungsung University, Busan 608-736, Korea Email address: jiny0610@hotmail.com

bepartment of Mathematics, Kyungsung University, Busan 608-736, Korea Email address: bslee@ks.ac.kr 